\title{
AN EXACT SOLUTION OF THE NONLINEAR DIFFERENTIAL EQUATION
}

$$
\ddot{y}+p(t) y=q_{m}(t) / y^{2 m-1}
$$

\section{JAMES L. REID}

ABSTRACT. An exact solution of the nonlinear differential equation $\ddot{y}+p(t) y=q_{m}(t) / y^{2 m-1}$ is found to be $y=\left[u^{m}+c(m-1)^{-1} W^{-2} v\right]^{1 / m}$ if $q_{m}(t)=c(u v)^{m-2} \cdot u$ and $v$ are independent solutions of $\ddot{y}+p(t) y=0$ and $W$ is their Wronskian.

E. Pinney [1] has shown that the nonlinear differential equation

$$
\ddot{y}+p(t) y=c / y^{8}
$$

has exact solutions of the form

$$
y=\left[u^{2}+c W^{-2} v^{2}\right]^{1 / 2}
$$

when $y\left(t_{0}\right)=y_{0} \neq 0$ and $\dot{y}\left(t_{0}\right)=\dot{y}_{0}$, for $c$ an arbitrary constant and $p(t)$ given. The functions $u$ and $v$ are independent solutions of the linear equations

$$
\ddot{y}+p(t) y=0
$$

for which $u\left(t_{0}\right)=y_{0}, \dot{u}\left(t_{0}\right)=\dot{y}_{0}, v\left(t_{0}\right)=0, \dot{v}\left(t_{0}\right) \neq 0$, where their Wronskian $W=u \dot{v}-v \dot{u}=$ const $\neq 0$.

With $m$ real and finite, and $m \neq 0,1$, it is possible to show that

$$
y=\left[u^{m}+c(m-1)^{-1} W^{-2} v^{m}\right]^{1 / m}
$$

is an exact solution of

$$
\ddot{y}+p(t) y=q_{m}(t) / y^{2 m-1}
$$

provided that $u$ and $v$ remain independent solutions of (3) and subject to the same conditions above, except that $v_{0}$ need not be zero, and provided that

$$
q_{m}(t)=c(u v)^{m-2} .
$$

The proof is simple and will be omitted.

Although $q_{m}(t)$ clearly restricts the general class of nonlinear equations implied by (5), important physical problems occur with

Received by the editors April 14, 1970.

AMS 1969 subject classifications. Primary 1302, 1304; Secondary 7034, 7834.

Key words and phrases. Exact solution, nonlinear differential equations, homogeneous nonlinear equation, initial conditions.

Copyright @ 1971, American Mathematical Society 
initial conditions such as to make the solution (4) physically interesting. Moreover, it is interesting to regard the use of $u v$ in $q_{m}(t)$ as a method for generating nonlinear differential equations, for which an exact solution is (4). The arbitrary choice of $p(t)$ allows a wide range of possibilities. Taking $p(t)= \pm \omega^{2}=$ const provides two immediate examples:

$$
\begin{aligned}
& \ddot{y}+\omega^{2} y=c_{1}(\sin \omega t \cos \omega t)^{m-2} / y^{2 m-1}, \\
& \ddot{y}-\omega^{2} y=c_{1} / y^{2 m-1},
\end{aligned}
$$

having solutions

$$
\begin{aligned}
& y=\left[a^{m} \cos ^{m} \omega t+c_{m} b^{m} \sin ^{m} \omega t\right]^{1 / m}, \\
& y=\left[a^{m} e^{m \omega t}+c_{m}^{\prime} b^{m} e^{-m \omega t}\right]^{1 / m}
\end{aligned}
$$

respectively, where

$$
c_{m}=c_{1} /\left[\omega^{2}(a b)^{m}(m-1)\right] \text { and } c_{m}^{\prime}=c_{1} /\left[4 \omega^{2}(a b)^{m}(m-1)\right] .
$$

Constants $a$ and $b$ are determined by the initial conditions, while $c_{1}$, equal to $c(a b)^{m-2}$, is essentially arbitrary.

\section{REFERENCES}

1. E. Pinney, The nonlinear differential equation $y^{\prime \prime}+p(x) y+c y^{-3}=0$, Proc. Amer. Math. Soc. 1 (1950), 681. MR 12, 336.

Clemson University, Clemson, South Carolina 29631 